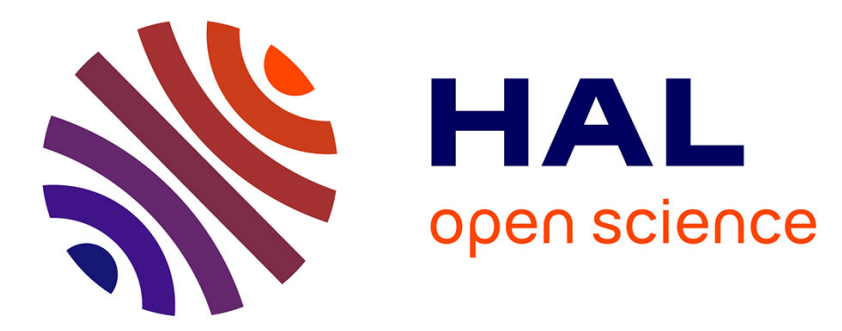

\title{
Quantum Hall effect and Fermi surface instabilities in (TMTSF) 2ClO4
}

M. Ribault, J. Cooper, D. Jérome, D. Mailly, A. Moradpour, K. Bechgaard

\section{To cite this version:}

M. Ribault, J. Cooper, D. Jérome, D. Mailly, A. Moradpour, et al.. Quantum Hall effect and Fermi surface instabilities in (TMTSF) 2ClO4. Journal de Physique Lettres, 1984, 45 (19), pp.935-941. 10.1051/jphyslet:019840045019093500 . jpa-00232434

\section{HAL Id: jpa-00232434 https://hal.science/jpa-00232434}

Submitted on 1 Jan 1984

HAL is a multi-disciplinary open access archive for the deposit and dissemination of scientific research documents, whether they are published or not. The documents may come from teaching and research institutions in France or abroad, or from public or private research centers.
L'archive ouverte pluridisciplinaire HAL, est destinée au dépôt et à la diffusion de documents scientifiques de niveau recherche, publiés ou non, émanant des établissements d'enseignement et de recherche français ou étrangers, des laboratoires publics ou privés. 


\title{
Quantum Hall effect and Fermi surface instabilities in (TMTSF) $)_{2} \mathrm{ClO}_{4}$
}

\author{
M. Ribault, J. Cooper (*), D. Jérome, D. Mailly $\left({ }^{* *}\right)$, A. Moradpour and K. Bechgaard $\left(^{+}\right)$ \\ Laboratoire de Physique des Solides, Bâtiment 510, Université Paris Sud, 91405 Orsay Cedex, France
}

(Reçu le 2 mai 1984, accepté le 17 août 1984)

\begin{abstract}
Résumé. - Nous avons observé des pics de magnétorésistance au même champ magnétique que les marches de l'effet Hall dans le composé (TMTSF) ${ }_{2} \mathrm{ClO}_{4}$. Les mesures ont été réalisées entre $0,1 \mathrm{~K}$ et $1,2 \mathrm{~K}$ dans des champs magnétiques allant jusqu'à $8,3 \mathrm{~T}$, sur un échantillon ayant un rapport de résistivité supérieur à 1600 . Ces résultats sont discutés dans un modèle associant l'effet Hall quantique et les transitions de phase.
\end{abstract}

\begin{abstract}
Peaks in magnetoresistance at the same magnetic field as the steps in the Hall voltage are observed in (TMTSF) ${ }_{2} \mathrm{ClO}_{4}$. These measurements were performed between 0.1 and $1.2 \mathrm{~K}$, in magnetic fields up to $8.3 \mathrm{~T}$, on a sample having a resistivity ratio $R(T=300 \mathrm{~K}, H=0) / R(T \rightarrow 0$, $H \rightarrow 0)>1600$. These results are discussed within a model associating the quantum Hall effect with phase transitions.
\end{abstract}

\section{Introduction.}

(TMTSF) ${ }_{2} \mathrm{X}$ is a family of salts built from the organic cation (TMTSF) ${ }^{+}$and a variety of mineral anions $\left(\mathrm{PF}_{6}\right)^{-},\left(\mathrm{AsF}_{6}\right)^{-},\left(\mathrm{ReO}_{4}\right)^{-},\left(\mathrm{ClO}_{4}\right)^{-},\left(\mathrm{NO}_{3}\right)^{-} \ldots$ At room temperature these salts are electrical conductors with a metallic behaviour [1]; at low temperature they exhibit a wide variety of ground states : superconductors [2], magnetic insulators [3], anion-order-induced insulators [4], depending on the anion, the pressure and the cooling rate. (TMTSF) ${ }_{2} \mathrm{ClO}_{4}$ is one of the most fascinating : at zero pressure, when slowly cooled, it undergoes a structural phase transition

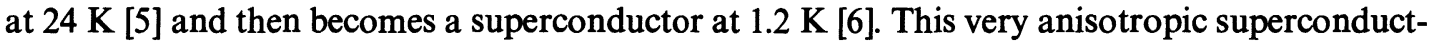
ing state $[7,8]$ condenses from an open, quasi-one-dimensional, Fermi surface (FS) [9]. The latter is restored by magnetic fields greater than the upper critical field $H_{\mathrm{c} 2}$. However when the magnetic field along the $\mathrm{c}^{*}$ direction is increased further this FS collapses probably in a series of steps [10-12]. The high field states are magnetic [13] and their existence was suggested to result from a quasi2D FS associated with either excitonic [10] or orbitally [12] induced phase transitions. We present in this paper magnetoresistance (MGR) and Hall voltage $(V H)$ measurements performed on a very high quality sample after cooling at the standard slow rate (4 hours from $35 \mathrm{~K}$ to $4.2 \mathrm{~K}$ ) [13]. These results, besides showing clearly first-order phase transition behaviour, reinforce strongly

(*) Institute of Physics of the University, Bijenička Cesta 46, POB 304, 41001 Zagreb, Yugoslavia.

(**) CNET Bagneux, 196, rue de Paris, 92220 Bagneux, France.

$\left({ }^{+}\right)$H. C. Oersted Institute, DK 2100 Copenhagen, Denmark. 
the "Quantum Hall Effect " (QHE) character of the magnetotransport properties; they are of critical interest for the discussion of the high magnetic field electronic states $[17,22]$.

\section{Experimental details.}

Since our previous paper was published [10], four new samples have been studied both in the lowfield and in the high-field regimes. The lengths $(L)$ along a ranged from 2.6 to $7 \mathrm{~mm}$, the widths

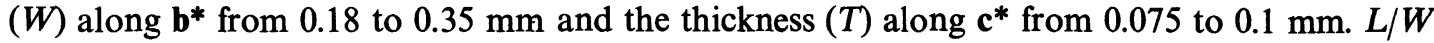
ratios varied from 9 to 28 . On these various samples, the results are qualitatively the same; we report here the results obtained, in high field, on our best sample, in the slowly cooled state; effects of the cooling rate will be reported in a forthcoming paper [14].

Great care has been taken to align the sample properly and therefore we estimate that the magnetic field is perpendicular to a within $2^{\circ}$ and lies along the $c^{*}$ direction within $6^{\circ}$.

The crystal dimensions were $\left(\mathbf{a} \times \mathbf{b}^{*} \times \mathbf{c}^{*}\right)=(7 \times 0.25 \times 0.075) \mathrm{mm}^{3}$ i.e. $L / W=28$. The electrical leads were $17 \mu \mathrm{m}$ diameter gold wires glued on to gold evaporated contacts with silver paint. Silver paint current contacts covered the ends and two pairs of « $V H$ contacts » (1-2) and (3-4) were at $1 / 3$ and $2 / 3$ of $L$ (see insert Fig. 1). The $V H$ contacts are used in a different arrangement $(1,3)$ and $(2-4)$ to monitor MGR voltage $V L$.

The measurements are performed using an A.C. technique with a current up to $100 \mu \mathrm{A}$ at $77 \mathrm{~Hz}$. All the results have been reconfirmed with a D.C. technique.

In this geometry, with such large $V L$ and $V H$, great care must be taken to avoid spurious effects. Both $V H$ and $V L$ are defined by combining measurements for opposite directions of the magnetic field, upward (u) and downward (d) (see note).

$$
V H=\left(V H_{\mathrm{u}}-V H_{\mathrm{d}}\right) / 2, \quad V L=\left(V L_{\mathrm{u}}+V L_{\mathrm{d}}\right) / 2 .
$$

On cooling the sample from room temperature no detectable jumps of electrical resistance were observed. Cooled from $35 \mathrm{~K}$ to $4.2 \mathrm{~K}$ in about 4 hours, the sample is in a state where neither NMR [13] nor resistivity [15] have detected magnetic domain effects.

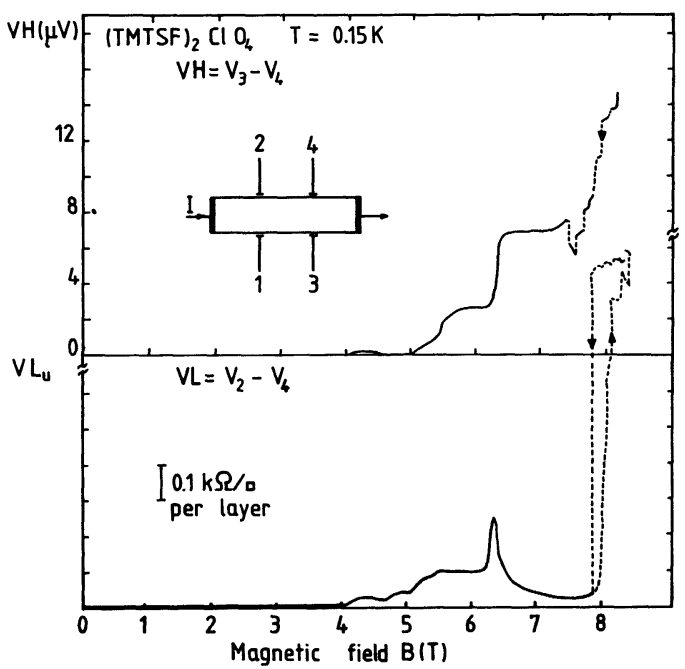

Fig. 1. - Insert : schematic arrangement of the contacts on the sample; the ends are covered with silver paint, the two pairs of Hall contacts are 1-2 and 3-4. Main figure : against the applied magnetic field are drawn the Hall voltage $V H$, as measured between 3 and 4, and the longitudinal voltage $V L_{u}$, as measured between 2 and 3 . 
The resistance ratio $R(T=300, H=0) / R(0,0)$ is at least 1600 (2 200 and 1600 on contacts 2-4 and $1-3$ respectively).

The critical magnetic field, defined at 0.9 of the normal resistance value is $0.095 \mathrm{~T}$ at $0.11 \mathrm{~K}$. In a magnetic field lower than $10^{-4} \mathrm{~T}$ the critical temperature is $1.19 \mathrm{~K}$ and the width of the transition is $0.08 \mathrm{~K}$.

\section{Experimental results.}

Figure 1 shows the results of the first measurements on this sample; $V L$ has been monitored mainly in increasing field whereas $V H$ was measured in decreasing field.

$V H$ is very similar to what has been observed in all samples in that there are two well defined steps respectively between 6.4 and $6.2 \mathrm{~T}$ and between 5.5 and $5.4 \mathrm{~T}$ and, in high field, $V H$ is electron like (note that this implies that at low field $V H$ is hole like); at the $6.2 \mathrm{~T}$ step $V H$ increases by a factor 2.5. The new result, for us (see [12]), is the (8.3-7.4 T) variation, from 8.3 to $7.4 \mathrm{~T}$ the variation is irreversible; $V H$ has not reached a saturation value in $8.3 \mathrm{~T}$; the increase at this step is 3 on $V H=\left(V_{2}-V_{1}\right)$ and more than 2 on $V H=\left(V_{3}-V_{4}\right)$. On $\left(V_{3}-V_{4}\right)$ (Fig. 1), just before the $7.4 \mathrm{~T}$ step, there is a drop of $20 \%$; such a drop at the beginning of a step was observed on several samples at the $6.2 \mathrm{~T}$ step; its occurrence will be discussed in [14]. In our previous work [10] only the beginning of the $7.4 \mathrm{~T}$ step was observed, at fields exceeding $8 \mathrm{~T}$; this may be due to both hysteresis problems and misorientation. A calculation of $\rho_{x y}$, the transverse resistivity of a molecular sheet gives $4.2 \mathrm{~K} \Omega$ /layer on the (6.4-7.4 T) plateau a value which is very close to $(1 / 6) \times\left(h / e^{2}\right)$. However it is really very difficult to make sense of the exact value of $V H$ as, from sample to sample, or for a given sample, from one pair of contacts to the other, the calculated $\rho_{x y}$ can vary by more than $25 \%$. This is due to at least two experimental problems : sample inhomogeneities which can modify the exact current density $(j)$ and an extension of the evaporated gold contact which can reduce the effective width $\left(W^{*}\right)$ of the sample $\left(\rho_{x y} \propto W^{*} / j\right)$. Up to now, $4 \mathrm{~K} \Omega$ appears to be the mean value obtained from all our samples for the (6.4-7.4 T) plateau. If the exact value of $\rho_{x y}$ cannot be determined very accurately, the ratio of the values of $V H$ on successive plateaus, for a fixed pair of contacts, is more reliable. At the lowest temperature [14] the value of $V H(6.5) / V H(6) / V H(5.4)$ extrapolates to $1 / 0.5 / 0.33$ whereas $V H(8.3) / V H(6.5)$ equals at least 2 to $3 / 1$.

In MGR (in fact, on Fig. 1, is shown $V L_{\mathbf{u}}$ ) new features appear. $V L$ shows minima at fields expected from Kajimura et al. [16] but extra peaks of MGR are displayed at magnetic fields just centred on the $V H$ steps. A very small peak was shown in [10] whose value, expressed as a variation of resistivity, is of the same order as the $6.3 \mathrm{~T}$ peak. The $7.8 \mathrm{~T}$ increase is very irreversible but we can see that the slope of the continuous sections of $V L(H)$ is negative at fields higher than 8.2 T. So three peaks are seen at respectively 5.4,6.3 and 8.2 $\mathrm{T}$. These peaks associated with the steps of $\mathrm{VH}$ are our main subject of interest in this paper. Among these peaks, only the one at 6.3 $\mathrm{T}$ is fully developed. Its main characteristic is that $V L$ is lower on the high side of the $V H$ step than it is on the low side and continues to decrease whereas $V H$ is constant; this will be discussed in the next part. The $8.2 \mathrm{~T}$ peak exhibits a very strong irreversibility; in decreasing fields the drop from the high field value occurs at $7.8 \mathrm{~T}$ in less than $0.02 \mathrm{~T}$. The irreversibility of the $6.3 \mathrm{~T}$ peak is less than $0.03 \mathrm{~T}$.

In figure 2, the temperature variation of both the 6.3 and $5.4 \mathrm{~T}$ peaks is shown. In the insert is $V L$ and on the main figure is $V L_{u}$ as previously defined; the difference, explained in the foot note, does not change the qualitative behaviour and comes down to an increase from $V L_{\mathbf{u}}$ to $V L$ by an amount varying from one side to the other of the peak; this amount is indicated by the arrows on figure 2 . The data in figure 1, figure 2 main, figure 2 insert were obtained respectively on the first, second (after warming up to $77 \mathrm{~K}$ ) and fourth (after warming up to $90 \mathrm{~K}$ ) cooling cycles; in spite of these thermal treatments, they are very close to each other. 


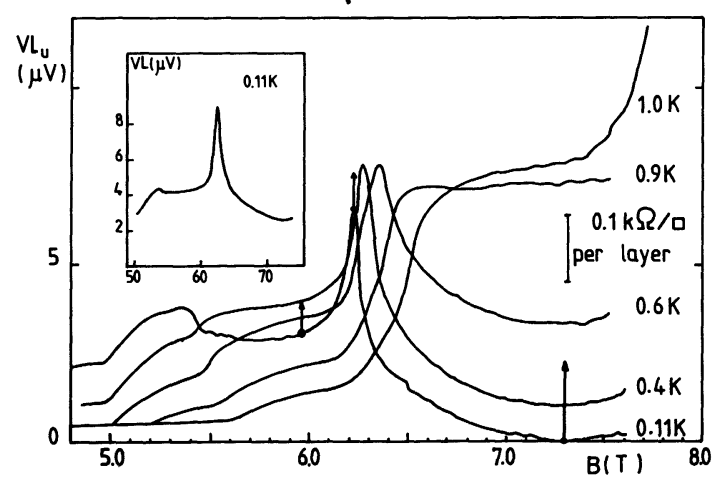

Fig. 2. - Against the applied magnetic field are drawn, in the insert, the correct longitudinal voltage $V L$ measured at $0.11 \mathrm{~K}$ and on the main figure the longitudinal voltage $V L_{u}$ as measured in upward magnetic field. The arrows indicate the translation to go from $V L_{\mathrm{u}}$ to $V L$.

We can see that, up to $0.6 \mathrm{~K}$, the $6.3 \mathrm{~T}$ peak is well marked, but that the decrease of $V L$, in high field, below its $5.9 \mathrm{~T}$ value does not persist at temperatures higher than $0.4 \mathrm{~K}$; this also corresponds to the temperature range where the (6.5-7.5 T) $\mathrm{VH}$ plateau becomes temperature dependent [10]. The shift to higher fields of all the characteristic features : peaks, oscillations and threshold field, can also be seen.

On the $0.9 \mathrm{~K}$ curve, between $6.8 \mathrm{~T}$ and 7.5 T, are oscillations of MGR, their frequency is $500 \mathrm{~T}$. The most striking behaviour of these oscillations is that their amplitudes decrease with both increasing and decreasing temperature, and that they do not exist at the lowest temperature; such a behaviour may be compared with the results of Chaikin et al. [12] in the 4 to $10 \mathrm{~K}$ temperature range in magnetic fields exceeding $10 \mathrm{~T}$. These oscillations seem to exist only in the intermediate temperature and magnetic field range when the step and plateaus pattern of $V H$ is not well established yet.

Let us now summarize the $0.11 \mathrm{~K}$ MGR measurements :

$$
R(0) / R(3.5 \mathrm{~T}) / R(6.3 \mathrm{~T}) / R(7.3 \mathrm{~T}) / R(8.3 \mathrm{~T})=1 / 2.2 / 80 / 22 / 300
$$

where $R(0)$ is the extrapolation to $H=0$ of the low field MGR.

\section{Discussion.}

In reference [10] two possible interpretations of the $V H$ plateaus were put forward. They were associated either with the quantum Hall effect as observed in two-dimensional electron systems or with a series of phase transitions induced by the magnetic field. Recently the latter viewpoint has been developed in an elegant paper of Gor'kov and Lebed' [17]. They show that for an open tight binding Fermi surface of the type expected to occur in (TMTSF) ${ }_{2} \mathrm{ClO}_{4}$ at low fields, the nesting, determined by the generalized susceptibility $X(Q, 0)$, will be a periodic function of the magnetic field. This leads to a series of phase transitions in spin density wave states with onset fields which are approximately periodic in $1 / H$.

On the other hand, the experimental results given in the previous section, in particular the observation of the minimum in MGR, the flatness of the plateaus in $V H$ and their amplitude now point even more strongly towards the QHE. In the discussion we wish to show that in fact these two points of view are not mutually exclusive and that it may be possible to combine them into a single consistent picture.

Firstly we will discuss how the QHE can occur in such systems. As mentioned before [10, 18], for $\mathrm{H} / / \mathrm{c}^{*}$ the band structure of (TMTSF) ${ }_{2} \mathrm{ClO}_{4}$ will become approximately two-dimensional when $\hbar \omega_{\mathrm{c}} \gg t_{\perp}$, where $\omega_{\mathrm{c}}$ is the cyclotron frequency for electron motion in closed orbits in the $a-b$ 
plane and $t_{\perp}$ is the tight binding transfer integral along the least conducting $\mathrm{c}$ direction. This condition means that in $k$ space the occupation of a given Landau level will not depend on $k_{c^{*}}$, so the system can be viewed as a stack of nearly decoupled 2D layers. Note that this is independent of whether electron transport along $\mathbf{c}^{*}$ is coherent or diffusive [19], but it does imply that the conductivity along the $\mathrm{c}^{*}$ axis is very small in these $(H, T)$ conditions. Furthermore we notice that, contrary to the QHE of inversion layers, the motion of the carriers within the $a-b$ plane of (TMTSF) ${ }_{2} \mathrm{ClO}_{4}$ in a high magnetic field departs strongly from isotropy. However we do not believe that this should significantly affect the overall picture given in this discussion.

In 2D inversion layers the « ordinary » QHE can be understood in the following simple way [20]. There are a large number of electrons in localized non conducting states which pin the Fermi level to a certain energy, and a smaller number of mobile electrons which occupy Landau levels whose $k$ space areas $A_{i}$ are quantized according to the usual conditions : $A_{i}=(2 \pi e H / c \hbar) \times(i-1 / 2)[21]$ where $i$ is an integer. Thus all the Landau levels below $E_{\mathrm{f}}$ are fully occupied and those above are empty. As $H$ is increased there is a transfer of electrons from the localized to the mobile states so that the Landau levels below $E_{\mathrm{f}}$ continue to be fully occupied as they expand. This means that the number of mobile carriers increases linearly with $H$ and then suddenly decreases by an amount corresponding to the degeneracy of one Landau level. $V H=(H I / n e W)$ thus has a series of steps given by $\left(h / i e^{2}\right)$. Thus a necessary condition for the ordinary QHE is to have a reservoir of nonconducting electrons which enables the Landau levels of the conducting electrons to remain completely full over an extended field range. We wish to show that a reservoir can be found in a more convenient way in (TMTSF) ${ }_{2} \mathrm{ClO}_{4}$ than it is usually in heterojunctions.

In our work the $\mathrm{VH}$ plateaus appear above the threshold field, when the (TMTSF) ${ }_{2} \mathrm{ClO}_{4}$ has undergone a spin density wave (SDW) transition. In this high field state the conduction band is split by SDW gaps into a low lying part which forms a " condensate " of electrons not contributing to the electrical conduction and a high energy part which gives rise to the electronic transport properties. Both nesting vector $Q$ and SDW amplitude can be magnetic field dependent and so, in the high field state, can induce Fermi surface variations. Then electrons may be transferred from a reservoir : the " condensate", to the free carriers and back in such a way that the Landau levels are either fully occupied or completely empty. Continuous variations in the number of carriers can give rise to plateaus and discontinuous variations in steps in $V H$. When these variations occur, $V H$ is quantized in $\left(h / e i^{2}\right)$ if all the Landau levels are fully occupied, but the values of $i$ are not necessarily successive integers and the values of $H$ where $i$ changes are not necessarily periodic in $(1 / H)$. At each step, these Fermi surface variations change the reference of the normal $V H$ thus, contrary to the ordinary QHE, plateaus and steps of $V H$ do not vary about a linear $V(H)$ variation. So we have outlined a picture in which the $V H$ quantization may be viewed as arising from a series of phase transitions but in these phases the Landau levels can be fully occupied over an extended field range. This origin may induce a certain number of differences with the " ordinary " QHE. A somewhat similar interpretation of our experimental results has been developed independently in a quantitative theory by Heritier et al. [22].

Whereas the standard QHE, given by heterojunctions, only one type of carrier is under consideration, in the Fermi surface variations picture it is possible to have Hall quantization from electron and hole Landau levels simultaneously as long as both are fully occupied. In the case of equal number of electrons and holes, which corresponds for example to $Q=(\pi / 2 a, 0,0)$, the plateaus of $V H$ seem difficult to understand; if $Q$ is incommensurate, then the electron and hole FS are not equal and we deal with three systems : electrons, holes and "condensate ". Now, let us go back to our experimental results.

The $V H$ plateaus, the peaks in MGR associated with the steps of $V H$, the opposite variation of $V H$ and MGR in going through the (6.2-7.4 T) field range are signs of an electronic process which is close to the QHE. But on the (6.5-7.5 T) plateau the MGR does not decrease by orders of magnitude and that could work against QHE except if one keeps in mind that : i) defects (cracks, 
inhomogeneities, SDW domains) [14] can prevent us from observing the peak [10, 12]; ii) there are complications because of the absence of cubic symmetry; iii) some conductivity in the c direction can reduce the $\rho_{x x}$ drop. Consideration of $V H$ or MGR, whenever we discuss the numerical values, leads to problems, but variations with $H$ and $T$ are significant. As discussed above, at the lowest temperature, in good samples, $V H(6.5) / V H(6) / V H(5.4)=1 / 0.5 / 0.33$ whereas here $V H(8.3) / V H(6.5)=2$ to 3 and $V H(8.3)$ has not yet reached a plateau.

In a first approach, we consider the lowest magnetic field plateaus (below $7.4 \mathrm{~T}$ ) for which the non-standard character of the QHE is not trivial. Considering the amplitudes ratios as successive integers, they correspond to 1, 2, 3 fully occupied Landau levels, but considering their position in $H$ they would correspond to 7, 8, 9 fully occupied Landau levels and from the exact value of $V H$ to $6,12,18$ fully occupied Landau levels. In the last approach we have to consider spin and valley degeneracy of the conduction electrons; to do that we need to know the nesting conditions... But the discrepancy between the position of the steps and the amplitude of $V H$ will remain except if the steps are due to contributions of non equivalent pockets (electron pockets); then we are not dealing any more with the standard QHE. Considering the small hysteresis measured here, as well as in [16], the development of steps and plateaus without any overall linear $V(H)$ law, our picture of QHE associated with Fermi surface variations may apply to these low field plateaus as well as to the high field plateau. The Gor'kov and Lebed' paper [17] is important because it demonstrates the instability of the electronic system, but within this model the instabilities would have been either more spaced (let us say one at $10 \mathrm{~T}$, another at $\therefore .4 \mathrm{~T}$ ) or closer to the $1 / \mathrm{H}$ periodicity.

Another important point is the temperature variation of steps, plateaus and connected features. In ordinary QHE, when increasing temperature, the variations are softened but always occur at the same magnetic field. In our system, as is usual for phase transitions, the onset fields are temperature dependent and features connected with gap opening can be observed. They are obvious in the (6.2-7.5 T) range when the temperature is increased $: \mathrm{i}) \mathrm{VH}$ decreases, ii) MGR increases, iii) high frequency oscillations of MGR are observed in a small temperature range. This is easy to understand considering that, when gaps are not fully developed, the Fermi level can cross other valleys and/or the carriers can be thermally excited and this can occur only in a small temperature range [23].

\section{Conclusion.}

We report, for the first time, $V H$ and MGR measurements for an organic conductor that can be understood within the quantum Hall effect framework. We demonstrate that a simple QHE approach is inadequate to explain our results and we suggest a picture associating the quantum Hall effect i.e. fully occupied Landau levels with variations of the nesting of the Fermi surface.

\section{Acknowledgments.}

We would like to acknowledge our colleagues L. Brossard, S. Tomic, F. Creuzet for constructive discussions. Their remarks improved the coherence of this paper. We also like to thank $\mathbf{M}$. Héritier, P. Lederer, G. Montambaux for their stimulating interest during the conclusion of this work. The development of their theory was a great encouragement [22]. We cannot forget earlier discussions with K. Murata, J. Tuchendler and C. Weyl.

\section{Note.}

It is well known that misalignment of the Hall voltage contacts introduces in $\mathrm{VH}$ some contribution of MGR that can be suppressed by subtracting measurements with the magnetic field reversed. But it was less obvious that our geometry (Fig. 1 insert) introduces a Hall contribution in magnetoresistance voltage $V L$. However this can be shown very easily. 
The longitudinal voltage can be measured either as $\left(V_{1}-V_{3}\right)$ or as $\left(V_{2}-V_{4}\right)$ and the transverse voltage either as $\left(V_{1}-V_{2}\right)$ or $\left(V_{3}-V_{4}\right)$. $V_{1}-V_{3}=\left(V_{1}-V_{2}\right)+\left(V_{2}-V_{4}\right)+$ $\left(V_{4}-V_{3}\right)$.

This elementary relation can be written as : $\left(V_{1}-V_{3}\right)-\left(V_{2}-V_{4}\right)=\left(V_{1}-V_{2}\right)-$ $\left(V_{3}-V_{4}\right)$; the difference between longitudinal voltages is equal to the difference between transverse voltages. The difference between transverse voltages contains not only some contribution of MGR but also, as pointed out in part 3, differences between the $V H$ of the two pairs of contacts. Then, in some cases, the longitudinal voltage may contain an important contribution of $V H . V H$ is odd in magnetic field; thus, to obtain the correct magnetoresistance, we must add measurements with magnetic field reversed.

\section{References}

[1] Bechgaard, K., Jacobsen, C. S., Mortensen, K., Pedersen, H. J. and Thorup, N., Solid State Commun. 33 (1980) 1119.

[2] Jérome, D., Mazaud, A., Ribault, M. and Bechgaard, K., J. Physique Lett. 41 (1980) L-95.

[3] Scott, J. C., Pedersen, H. J. and Bechgaard, K., Phys. Rev. Lett. 45 (1980) 2125.

[4] Parkin, S. S. P., Jérome, D. and Bechgaard, K., Mol. Cryst. Liq. Cryst. 79 (1982) 213.

[5] Tomic, S., Jérome, D., Monod, P. and BechgaArd, K., J. Physique Lett. 43 (1982) L-839. Garoche, P., Brusetti, R. and BechgaArd, K., Phys. Rev. Lett. 49 (1982) 1346.

Pouget, J. P., Shirane, G., Bechgaard, K. and Fabre. J. M., J. Phys. B 27 (1983) 5203.

[6] Bechgaard, K., Carneiro, K., Olsen, M., Rasmussen, F. and Jacobsen, C. S., Phys. Rev. Lett. 46 (1981) 852.

[7] Choi, M., Chaikin, P. M., Huang, S. Z., Haen, P. and Greene, R. L., Phys. Rev. B 25 (1982) 6208.

[8] Mailly, D., Ribault, M., Bechgaard, K., Fabre, J. M. et Giral, L., J. Physique Lett. 43 (1982) L-711.

[9] Grant, P., Phys. Rev. B 26 (1980) 6888.

Grant, P., J. Physique Colloq. 44 (1983) C3-783.

[10] Ribault, M., Jérome, D., Tuchendler, J., Weyl, C. et BechgaArd, K., J. Physique Lett. 44 (1983) L-953.

[11] Garoche, P., Brusetti, R., Jérome, D. et Bechgaard, K., J. Physique Lett. 43 (1982) L-147.

[12] Chaikin, P. M., Mu Yong Chai, Kwak, J. F., Brooks, J. S., Martin, K. P., Noughton, M. J., Engler, E. M. et Greene, R. L., Phys. Rev. Lett. 51 (1983) 2333.

[13] Takahashi, T., Jérome, D., Bechgaard, K., J. Physique Colloq. 44 (1983) C4-805.

[14] Ribault, M., Cooper, J., Mailly, D., Moradpour, A. et Bechgaard, K., to be published.

[15] Ribault, M., J. Physique Colloq. 44 (1983) C3-827.

[16] Kajimura, K., Tokumoto, H., Tokumoto, M., Murata, K., Ukachi, T., Anzai, H., Ishigura, T. et SaIto, G., Solid State Commun. 44 (1982) 1573 and J. Physique Colloq. 44 (1983) C3-1059.

[17] Gor'kov, L. P., Lebed', A. G., J. Physique Lett. 45 (1984) L-433.

[18] Chaikin, P., Choi, M. J., Greene, R. L., J. Physique Coll. 44 (1983) C3-1067.

[19] CoOper, J., Weger, J. R., Delplanque, M., Jérome, D. et BechgaArd, K., J. Physique Lett. 37 (1976) L-349.

[20] Aoki, H. and Kaminura, H., Solid State Commun. 21 (1977) 45.

[21] Gold, A. V., The de Haas-van-Alphen effect in Sol. St. Phys. 1 Electrons in Metals (Gordon and Breach) 1968.

[22] Heritier, M., Montambaux, G. and Lederer, P., J. Physique Lett. 45 (1984) L-943 (this issue).

[23] Gor'Kov, L. and LEBED', A., to be published in Molecular crystals and liquid crystals, proceedings of ICSM 84, Abano Terme, June 1984. In this conference Gor'kov has shown that modifications of the superstructure, before the establishment of the $T=0$ ground state gaps, give rise to an intermediate region in $(H, T)$ space where such oscillations of magnetoresistance are expected. 